\title{
BMJ Open Validation of a non-invasive method for the early detection of metabolic syndrome: a diagnostic accuracy test in a working population
}

\author{
Manuel Romero-Saldaña, ${ }^{1}$ Pedro Tauler, ${ }^{2,3}$ Manuel Vaquero-Abellán, ${ }^{4}$ \\ Angel-Arturo López-González, ${ }^{5}$ Francisco-José Fuentes-Jiménez, ${ }^{6,7}$ \\ Antoni Aguiló, ${ }^{3,8}$ Carlos Álvarez-Fernández, ${ }^{1}$ Guillermo Molina-Recio, ${ }^{9}$ \\ Miquel Bennasar-Veny ${ }^{3,8}$
}

To cite: Romero-Saldaña M, Tauler P, Vaquero-Abellán M, et al. Validation of a noninvasive method for the early detection of metabolic syndrome: a diagnostic accuracy test in a working population. BMJ Open 2018;8:e020476. doi:10.1136/ bmjopen-2017-020476

- Prepublication history for this paper is available online. To view these files, please visit the journal online (http://dx.doi. org/10.1136/bmjopen-2017020476).

Received 13 November 2017 Revised 30 August 2018 Accepted 4 September 2018

Check for updates

(c) Author(s) (or their employer(s)) 2018. Re-use permitted under CC BY-NC. No commercial re-use. See rights and permissions. Published by BMJ.

For numbered affiliations see end of article.

Correspondence to

Dr Pedro Tauler;

pedro.tauler@uib.es

\section{ABSTRACT}

Objectives A non-invasive method for the early detection of metabolic syndrome (NIM-MetS) using only waist-toheight ratio (WHtR) and blood pressure (BP) has recently been published, with fixed cut-off values for gender and age. The aim of this study was to validate this method in a large sample of Spanish workers.

Design A diagnostic test accuracy to assess the validity of the method was performed.

Setting Occupational health services.

Participants The studies were conducted in 2012-2016 on a sample of 60799 workers from the Balearic Islands (Spain).

Interventions The NCEP-ATP III criteria were used as the gold standard. NIM-MetS has been devised using classification trees (the $\chi^{2}$ automatic interaction detection method).

Main outcome measures Anthropometric and biochemical variables to diagnose MetS. Sensitivity, specificity, validity index and Youden Index were determined to analyse the accuracy of the diagnostic test (NIM-MetS).

Results With regard to the validation of the method, sensitivity was $54.7 \%$, specificity $94.9 \%$ and the Validity Index $91.2 \%$. The cut-off value for WHtR was 0.54 , ranging from 0.51 (lower age group) to 0.56 (higher age group). Variables more closely associated with MetS were WHtR (area under the curve (AUC) $=0.85 ; 95 \% \mathrm{Cl} 0.84$ to 0.86 ) and systolic $\mathrm{BP}$ ( $\mathrm{AUC}=0.79 ; 95 \% \mathrm{Cl} 0.78$ to 0.80$)$ ). The final cut-off values for the non-invasive method were WHtR $\geq 0.56$ and $B P \geq 128 / 80 \mathrm{~mm} \mathrm{Hg}$, which includes four levels of MetS risk (very low, low, moderate and high).

Conclusions The analysed method has shown a high validity index (higher than $91 \%$ ) for the early detection of MetS. It is a non-invasive method that is easy to apply and interpret in any healthcare setting. This method provides a scale of MetS risk which allows more accurate detection and more effective intervention.

\section{INTRODUCTION}

The obesity epidemic which currently affects the world population has resulted in a

\section{Strengths and limitations of this study}

- This is the first study assessing the validation of a non-invasive method for the early detection of metabolic syndrome (NIM-MetS).

- A diagnostic test study has been carried out in a large sample of healthy workers.

- MetS was ascertained by using the National Cholesterol Education Program Adult Treatment Panel III (NCEP-ATP III) definition, but there is a lack of consensus regarding the definition of MetS.

- A new procedure to measure MetS using variables with universal cut-off points (waist-to-height ratio and blood pressure) is suggested.

- The NIM-MetS method has shown high specificity, but low sensitivity.

general increase in the prevalence of metabolic syndrome (MetS) ${ }^{1-3}$ Overweight and obesity are factors related to the onset of type 2 diabetes, hypertension, dyslipidaemia and cardiovascular diseases (CVDs). In particular, central obesity, which is defined as an excessive accumulation of abdominal fat, is an important predictor of cardiovascular risk and MetS. ${ }^{45}$ Metabolic syndrome is defined as a pluripathological state characterised by the joint presence of several cardiovascular risk factors such as abdominal obesity, high blood pressure (BP), and altered glucose and lipid metabolism (low high-density lipoprotein (HDL)-cholesterol and high triglycerides). ${ }^{6}$

Although there are several analytical/ instrumental techniques for measuring the amount and distribution of body fat, there is no consensus on the ideal method to calculate central adiposity, nor on how to decide which cut-off points provide greater accuracy, efficiency, sensitivity (S) and specificity (SP) in all cases. ${ }^{78}$ 
A simple and inexpensive alternative to these instruments as a way of quantifying abdominal fat is to make anthropometric measurements of central obesity. ${ }^{9}$ Waist circumference (WC), body mass index (BMI), waist-toheight ratio (WHtR), waist-to-hip ratio, hip-to-height ratio), Body Adiposity Index (BAI), Visceral Adiposity Index, A Body Shape Index (ABSI) and percentage of body fat $(\% \mathrm{BF})$ are some examples that can be found in numerous epidemiological studies, in which they try to indirectly relate intra-abdominal (visceral) fat with parameters such as morbidity and mortality, and also with prevalence of hypertension, diabetes, MetS, and so on. ${ }^{10-13}$

Since the mid-1990s, WHtR has been the most widely used anthropometric indicator and the one which has obtained the best predictive results for cardiovascular risk. ${ }^{14}$ In a previous publication, a non-invasive method for early detection of MetS (NIM-MetS) using only two anthropometric variables ( $\mathrm{WHtR}$ and $\mathrm{BP}$ ) has been proposed and validated. ${ }^{15}$ This method suggests WHtR $\geq 0.55$ as the predictive threshold for the early detection of MetS for both men and women and, also, for any age stratum.

The aim of this study is to validate the NIM-Mets method in a large representative sample of Spanish workers, to determine its predictive ability and to find out the stability of the cut-off value of WHtR $\geq 0.55$ by gender and age.

\section{MATERIAL AND METHODS Design and sample}

A diagnostic test using a cross-sectional study was carried out on a working population from the Balearic Islands (Spain) between 2012 and 2016. Subjects participating in the study were randomly selected during their work health periodic assessments. Every day, each worker was assigned a number and half of the examined workers were randomly selected using a random number table. A total of 69581 workers were invited to participate in the study. However, 8782 (12.6\%) refused to participate and, thus, the final number of participants was 60799 workers (10.2\% of the active population) belonging to different economic sectors (public administration, health services, etc), aged $20-70$ years; $57.3 \%$ of the participants was male $42.7 \%$ female.

Participants were informed of the purpose of the study before they provided written informed consent to participate. The study protocol complied with the Declaration of Helsinki for conducting medical research involving human subjects.

\section{Data collection and definition of variables}

To carry out the anthropometric measurements, recommendations contained in the manual 'International Standards for Anthropometric Assessment (ISAK)' were followed. ${ }^{16}$ All the measurements were made by specifically trained staff in order to minimise the variation coefficients. Each measurement was performed three times, taking the average as the final value.

The independent variables were classified into the following categories:

- Personal and health habits: gender, age and tobacco consumption.

- Anthropometric measurements:

- WC in $\mathrm{cm}$.

- BMI, calculated as body weight ( $\mathrm{kg}$ ) divided by height $(\mathrm{m})$ squared, in $\mathrm{kg} / \mathrm{m}^{2}$.

- \%BF calculated according to the Deurenberg equation: $\% \mathrm{BF}=1.2 \times(\mathrm{BMI})+0.23 \times($ age in years $)-10.8$ $\times$ (gender) -5.4 . Gender: female $(0)$, male $(1)$

- Waist-to-height ratio (WHtR), calculated as WC divided by height, both in $\mathrm{cm}$.

- ABSI calculated as WC/ $\left.\left((\mathrm{BMI})^{2 / 3} \text { (height }\right)^{1 / 2}\right)$.

Blood measurements:

- Systolic BP (SBP) in mm Hg.

- Diastolic BP (DBP) in mm Hg.

- Total cholesterol $(\mathrm{mg} / \mathrm{dL})$, low-density lipoprotein; LDL-cholesterol (mg/dL), HDL-cholesterol $(\mathrm{mg} / \mathrm{dL})$, glucose $(\mathrm{mg} / \mathrm{dL})$ and triglycerides $(\mathrm{mg} /$ $\mathrm{dL})$.

Body weight was measured to the nearest $0.1 \mathrm{~kg}$ with an electronic scale (Seca 700 scale, Seca GmbH, Hamburg). Height was measured to the nearest $0.5 \mathrm{~cm}$ with a stadiometer (Seca 220 (CM) telescopic height rod for column scales, Seca GmbH, Hamburg). WC was measured halfway between the lower costal border and the iliac crest. The measurement was taken at the end of a normal expiration with the subject standing up, with their feet together and their arms hanging down by their sides.

Venous blood samples were taken from the antecubital vein in suitable vacutainers without anticoagulant in order to obtain serum. The blood samples were taken after a 12-hour overnight fast. Participants sat and rested for at least $15 \mathrm{~min}$ before the blood samples were taken. Serum was obtained after centrifugation $\left(15 \mathrm{~min}, 1000 \times \mathrm{g}, 4^{\circ} \mathrm{C}\right.$ ) of the blood samples. The serum was stored at $-20^{\circ} \mathrm{C}$ and analyses were performed within 3 days. Concentrations of glucose, cholesterol and triglycerides were measured in serum following the standard procedures used in clinical biochemistry laboratories with an autoanalyser (SYNCHRON CXH9 PRO, Beckman Coulter, Brea, California, USA).

BP was determined after the subjects had rested in the supine position for $10 \mathrm{~min}$, using an automatic and calibrated sphygmomanometer (OMRON M3, OMRON Healthcare Europe, Spain). As in the case of the anthropometrical measurements, BP was measured three times, leaving a 1 min gap between each measurement, and the average value was then calculated.

Presence of MetS was ascertained by using the criterion suggested by the NCEP-ATP III definition (when three of five of the following characteristics are present, a diagnosis of metabolic syndrome can be made):

- Abdominal obesity (WC $\geq 102 \mathrm{~cm}$ in men and $\mathrm{WC}$ $\geq 88 \mathrm{~cm}$ in women). 
- Triglycerides $\geq 150 \mathrm{mg} / \mathrm{dL}$.

- HDL-cholesterol $<40 \mathrm{mg} / \mathrm{dL}$ in men and $<50 \mathrm{mg} / \mathrm{dL}$ in women.

- $\mathrm{BP} \geq 130 / 85 \mathrm{~mm} \mathrm{Hg}$.

- Fasting glucose $\geq 100 \mathrm{mg} / \mathrm{dL}$.

\section{Non-invasive method for early detection of MetS}

NIM-Mets is a new tool for screening for MetS based on the following anthropometric variables and cut-off values: WHtR $\geq 0.55$ and $\mathrm{BP} \geq 128 / 85 \mathrm{~mm} \mathrm{Hg}$.

This method classifies the population into two groups with different levels of risk:

- Workers with high risk of MetS (probability $>61.7 \%$ ): this group would contain those subjects with both positive variables, that is, $\mathrm{WHtR} \geq 0.55$ and $\mathrm{BP} \geq 128 / 85 \mathrm{~mm}$ $\mathrm{Hg}$.

- Workers with low risk of MetS (probability of $0.5 \%-$ $16.9 \%)$ : this group would contain those subjects who have any of the other possible combinations between the two variables considered.

\section{Statistical analysis}

Statistical analysis was carried out using IBM SPSS Statistics V.22.0 software (SPSS, IBM, Chicago, Illinois, USA) and Epidat V.4.2. (Department of Sanidade, Xunta de Galicia, Galicia, Spain). Continuous data are presented as mean values, SD and 95\% CI. Categorical data are shown as frequency counts and percentages. All the data were tested for their normal distribution (KolmogorovSmirnov test with Lilliefors adjustment).

Student's t-test and Mann-Whitney U test were used in the bivariate analyses for normal and non-normal distributed variables, respectively. Analysis of variance tests with the post hoc Bonferroni contrast method were carried out when more than two groups were considered in the analysis. The Levene test was used to determine variance equality. The $\chi^{2}$ test was applied to assess differences between groups in categorical variables.

Receiver operating characteristic (ROC) curves were performed and the area under the curve (AUC) was calculated to find which explanatory variables best predict the onset of MetS. We obtained the cut-off value for each explanatory variable through the Youden Index (JI) as JI $=\mathrm{S}+\mathrm{SP}-1$.

To measure the accuracy of the diagnostic test, S, SP, positive and negative predictive values (PPV and NPV), likelihood ratios (LH+ and LH-), Validity Index (VI) and JI were analysed. VI was calculated as the quotient between the sum of true positives and true negatives, divided by the total number of subjects, therefore representing the percentage of subjects properly classified by the test.

The modification of NIM-MetS was obtained from a clinical decision tree (classification) using the $\chi^{2}$ automatic interaction detection (CHIAD) technique as a growth method. The statistical significance level for splitting nodes and merging categories was $\mathrm{p}<0.05$, and significance values were corrected by the Bonferroni method, with a maximum number of iterations of 2000 .
The level of statistical significance was fixed in all the contrasts for an $\alpha$ error below $5 \%$, and the CIs were calculated with a $95 \%$ level of confidence.

\section{Patient and public involvement}

Patients were not involved in setting the research question and in the study design. All patients were randomly selected during their work health periodic assessments to participate in the study and they were interviewed face to face by trained researchers for detailed explanation of the purpose of this research and informed consent at the beginning. No patients were involved in data analysis or manuscript writing. Results of the research will not be disseminated to the patients.

\section{RESULTS}

\section{Characteristics of the study sample}

Of the 60799 workers, 34827 were male $(57.3 \%)$. The overall mean age was 40 years (39.9-40.1) (table 1). Among anthropometrical and blood parameters shown in table 1 , women showed higher \% BF and HDL-cholesterol values $(p<0.001)$, while men showed significantly higher values for the rest of the parameters shown in this table. The prevalence of smokers was $34.8 \%$ (36.6\% in men and $32.5 \%$ in women) and $17.6 \%$ of participants were obese (20.0\% men and $14.4 \%$ women). As regards to drug treatments, $6.6 \%$ of participants were undergoing antihypertensive treatment, 3.2\% lipid-lowering treatments and $1.7 \%$ antidiabetic treatments. Finally, the overall prevalence of MetS was $9.0 \%$, with $11.8 \%$ in men and $5.4 \%$ in women $(\mathrm{p}<0.001)$.

\section{NIM-MetS validation}

Table 2 shows the results of diagnostic tests after applying the NIM-Mets method compared with NCEP-ATP III as a control test. Overall, the indicators of the NIM-MetS method validation were as follows: $\mathrm{S}=54.7 \%$ (53.4-56.0), $\mathrm{SP}=94.9 \%(94.7-95.0)$ and $\mathrm{VI}=91.2 \%(91.0-91.5) . \mathrm{S}$ was higher in men $(59.4 \%)$ than in women $(40.9 \%)$.

As regards the NIM-MetS safety indicators, results in the total sample were: $\mathrm{PPV}=51.3 \%$ (50.0-52.6) and NPV $=95.5$ (95.3-95.7). By gender, PPV was higher in men (51.4\%) than in women $(50.8 \%)$, while NPV was higher in women $(96.7 \%)$ than in men $(94.5 \%)$. Finally, the overall JI was $0.50(0.48$ to 0.51$)$, higher in men (0.52) than in women (0.39).

A second question to be dealt with in this research was to compare the cut-off value for WHtR proposed by NIM-MetS with that obtained in the study sample, and thus determine its variability according to the gender variable and in different age groups (table 3 ). In the whole sample ( $\mathrm{n}=60799)$, a cut-off value of 0.54 was obtained for WHtR. In the group of men $(n=34827)$ the resulting threshold was 0.56 , while for women $(\mathrm{n}=25972)$ it was 0.53 .

It can be seen how the cut-off point increases with age. For men, it ranged from 0.55 (age 20-30 years) to 0.56 
Table 1 Characteristics of the sample according to gender

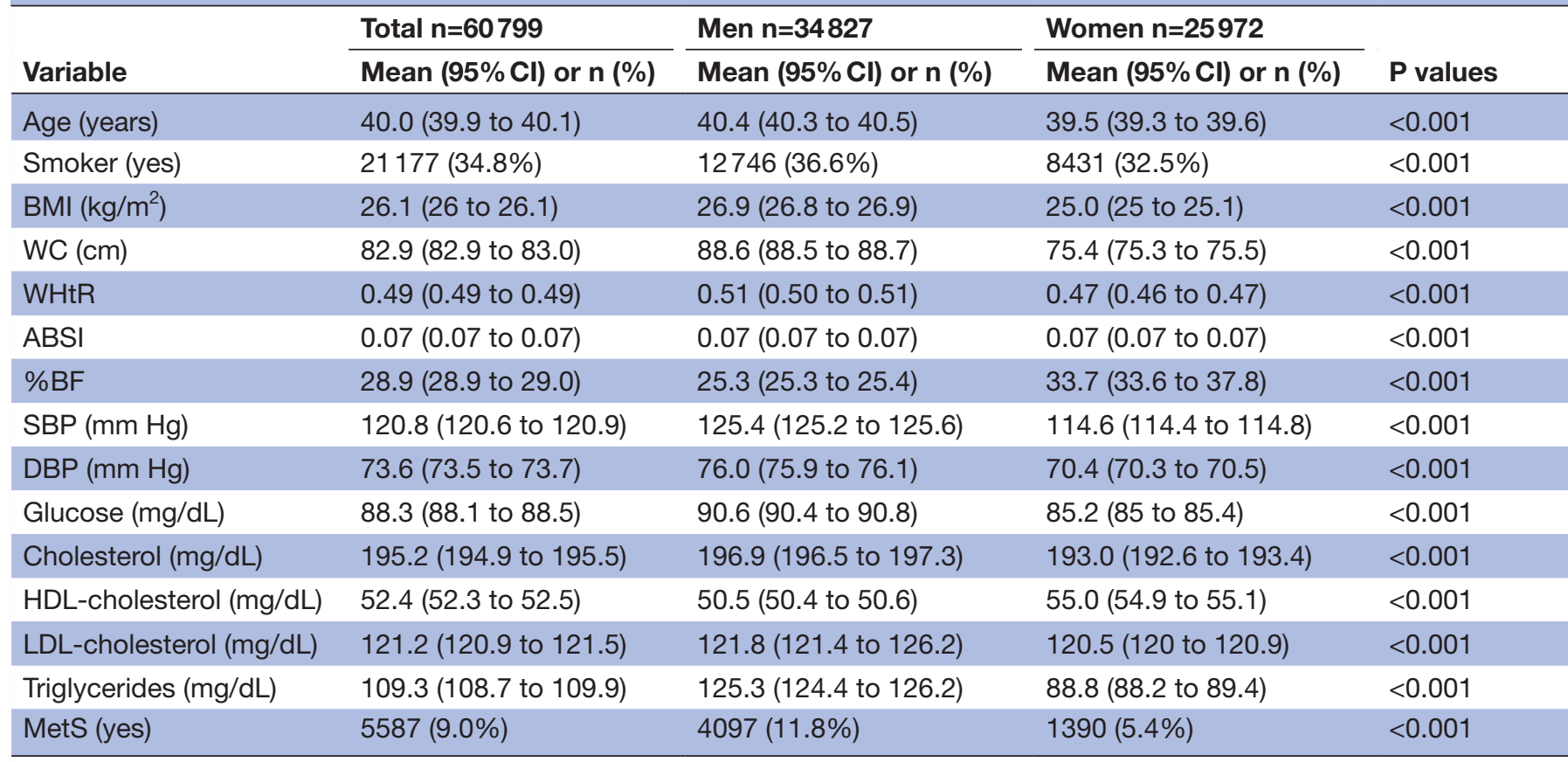

A value of $p<0.05$ indicates statistically significant differences between genders.

ABSI, A Body Shape Index; \%BF, percentage of body fat calculated according to the Deurenberg equation; BMI, body mass index; DBP, diastolic blood pressure; HDL, high-density lipoprotein; LDL, low-density lipoprotein; MetS, metabolic syndrome; SBP, systolic blood pressure; WC, waist circumference; WHtR, waist-to-height ratio.

(age $\geq 51$ years), whereas for women it was between 0.51 (age 20-30 years) and 0.55 (age $\geq 51$ years). The differences between the cut-off values for men and women become narrower as the age increased.

Figure 1 shows the results for the anthropometric variables' ROC curves. WHtR achieved the highest AUC 0.85 (95\% CI 0.84 to 0.86 ), with a cut-off value of 0.54 , reaching top values of $\mathrm{S}=68.5 \%, \mathrm{SP}=87.0 \%$ and $\mathrm{JI}=0.56$. The second variable with the highest AUC was WC, with $0.83(95 \%$ CI 0.82 to 0.84$)$, a cut-off value of $89.1 \mathrm{~cm}$ and $\mathrm{S}=72.5, \mathrm{SP}=77.6 \%$ and $\mathrm{JI}=0.5$. BMI with an $\mathrm{AUC}=0.80$ and $\mathrm{SBP}$ with $\mathrm{AUC}=0.79$ also stood out.

After that, different clinical decision trees were made with a range of cut-off values for WHtR and BP (table 4).

Table 2 Diagnostic test accuracy of the non-invasive method for early detection of metabolic syndrome against NCEP-ATP III

\begin{tabular}{|c|c|c|c|c|c|c|c|c|c|}
\hline & \multicolumn{9}{|c|}{ Standard reference NCEP APTIIII } \\
\hline & \multicolumn{3}{|l|}{ Total } & \multicolumn{3}{|c|}{ Men } & \multicolumn{3}{|c|}{ Women } \\
\hline & Yes & No & Total & Yes & No & Total & Yes & No & Total \\
\hline \multicolumn{10}{|l|}{ NIM-MetS (n) } \\
\hline Yes & 3001 & 2850 & 5851 & 2433 & 2300 & 4733 & 568 & 550 & 1118 \\
\hline No & 2486 & 52462 & 54948 & 1664 & 28430 & 30094 & 822 & 24032 & 24854 \\
\hline Total & 5487 & 55312 & 60799 & 4097 & 30730 & 34827 & 1390 & 24582 & 25972 \\
\hline \multicolumn{10}{|c|}{ Efficacy indicators, $95 \% \mathrm{Cl}$} \\
\hline Sensitivity & \multicolumn{3}{|c|}{54.7 (53.4 to 56.0$)$} & \multicolumn{3}{|c|}{59.4 (57.9 to 60.9$)$} & \multicolumn{3}{|c|}{40.9 (38.2 to 43.5$)$} \\
\hline Specificity & \multicolumn{3}{|c|}{94.9 (94.7 to 95.0$)$} & \multicolumn{3}{|c|}{92.5 (92.2 to 92.8 ) } & \multicolumn{3}{|c|}{97.8 (97.6 to 98.0$)$} \\
\hline PPV & \multicolumn{3}{|c|}{51.3 (50 to 52.6$)$} & \multicolumn{3}{|c|}{51.4 (50.0 to 52.8$)$} & \multicolumn{3}{|c|}{50.8 (47.8 to 53.8$)$} \\
\hline NPV & \multicolumn{3}{|c|}{95.5 (95 to 95.7$)$} & \multicolumn{3}{|c|}{94.5 (94.2 to 94.7$)$} & \multicolumn{3}{|c|}{96.7 (96.5 to 96.9$)$} \\
\hline VI & \multicolumn{3}{|c|}{91.2 (91.0 to 91.5$)$} & \multicolumn{3}{|c|}{88.6 (88.3 to 89.0$)$} & \multicolumn{3}{|c|}{94.7 (94.4 to 95.0$)$} \\
\hline $\mathrm{LH}+$ & \multicolumn{3}{|c|}{$10.6(10.2$ to 11.1$)$} & \multicolumn{3}{|c|}{$7.9(7.6$ to 8.3$)$} & \multicolumn{3}{|c|}{$18.3(16.5$ to 20.3$)$} \\
\hline LH - & \multicolumn{3}{|c|}{0.48 (0.46 to 0.49$)$} & \multicolumn{3}{|c|}{0.44 (0.42 to 0.46$)$} & \multicolumn{3}{|c|}{0.60 (0.58 to 0.63$)$} \\
\hline J & \multicolumn{3}{|c|}{0.50 (0.48 to 0.51$)$} & \multicolumn{3}{|c|}{$0.52(0.50$ to 0.53$)$} & \multicolumn{3}{|c|}{0.39 (0.36 to 0.41$)$} \\
\hline
\end{tabular}

JI, Youden Index; LH+, positive likelihood ratio; LH-, negative likelihood ratio; NIM-MetS, non-invasive method for early detection of metabolic syndrome; NPV, negative predictive value; PPV, positive predictive value; VI, Validity Index. 
Table 3 Area under the curve (AUC) and cut-off values for waist-to-height ratio (WHtR) according to gender and age groups

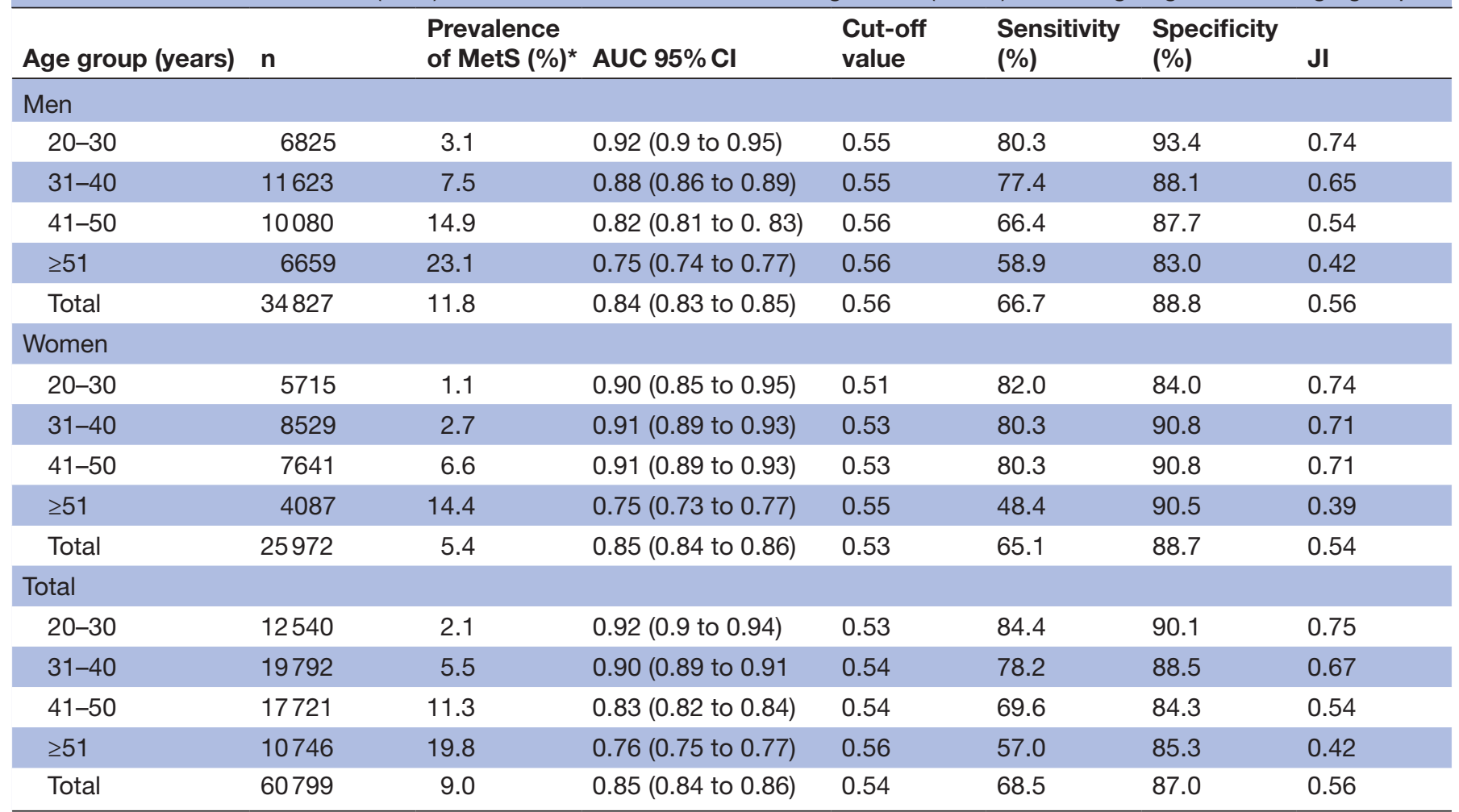

*MetS according to NCEP ATP III criterion.

$\mathrm{JI}$, Youden Index; MetS, metabolic syndrome.

Thus, the range of cut-offs for WHtR was defined by eight thresholds between 0.54 and 0.57 , and included, among others, the cut-off value proposed by NIM-MetS (WHtR $\geq 0.55$ ) and the cut-off value for the total sample (WHtR $\geq 0.54$ ). In addition, three models were established for BP: $\mathrm{BP} \geq 128 / 80 \mathrm{~mm} \mathrm{Hg}$ (cut-off values obtained for SBP and DBP as ROC curves, shown in figure 2); $\mathrm{BP} \geq 128 / 85 \mathrm{~mm}$ $\mathrm{Hg}$ (BP cut-off values proposed by NIM-MetS); and finally, only SBP $\geq 128 \mathrm{~mm} \mathrm{Hg}$ (second covariate with the highest adjusted OR in the multiple logistic regression). In this way, 24 clinical decision trees were set up using CHAID methodology. Each tree comprised of a parent node (node 0 ), two primary subsidiary nodes (nodes 1 and 2) and four secondary subsidiary nodes (nodes 3, 4, 5 and $6)$. Each of the last four nodes denoted the probability of having MetS. Thus, node 3 corresponds to the probability that a worker has MetS when both anthropometric variables are negative (below cut-off values). Node 4 indicates the probability that a worker has MetS when BP is above the cut-off value and WHtR is below. Node 5 represents the probability that a worker has MetS when BP is lower than the cut-off value and WHtR is above. Finally, node 6 shows the probability that a worker suffers from MetS when both variables are positive (above the cut-off values). The model $\mathrm{BP} \geq 128 / 80 \mathrm{~mm} \mathrm{Hg}$ was chosen because it had the highest JI value (greatest S and SP combined) at each of the WHtR cut-off points (table 4).

The next step was to select the final cut-off value for WHtR. To do this, the method's probability of detection (node 6 value) and the JI for the BP model chosen (BP $\geq 128 / 80 \mathrm{~mm} \mathrm{Hg}$ ) were plotted for each WHtR cut-off value (figure 2 ).

It was noted that the probability of detection of MetS in each tree (node 6 value) and the WHtR threshold, as well as the JI and the WHtR threshold, follow linear functions, in which the equations of its lines are as follows:

- Probability MetS (node 6$)=5.534 * \mathrm{WHtR}-2.58$

- $\mathrm{JI}=-1.758 * \mathrm{WHtR}+1.486$

Thus, the final threshold value for WHtR was determined by the cut-off points of both lines: $\mathrm{WHtR}=0.56$ (figure 2).

The resulting new method for the early detection of MetS (new NIM-MetS) includes these conditions: WHtR $\geq 0.56$ and BP $\geq 128 / 80 \mathrm{~mm} \mathrm{Hg}$. Figure 3 shows the decision tree created from these variables and cut-off points. The $\mathrm{S}$ of the proposed method was $56.4 \%$, SP was $94.5 \%$, VI was $91.1 \%$ and JI was 0.51 .

Finally, from the probabilities obtained in nodes 3, 4, 5 and 6, a risk gradient for MetS was developed, according to the WHtR and BP covariates and the proposed cut-off values. Thus, those subjects with lower $\mathrm{WHtR}$ and $\mathrm{BP}$ values than the cut-off point have a very low probability of suffering from MetS (PMetS=0.4\%). Low risk (PMetS=8.3\%) would be found only in those individuals with $\mathrm{BP}$ values over $128 / 80 \mathrm{~mm} \mathrm{Hg}$, but a low WHtR. A moderate level of risk (PMetS=16.3\%) would include normotensive subjects who had a WHtR $\geq 0.558$. Finally, 


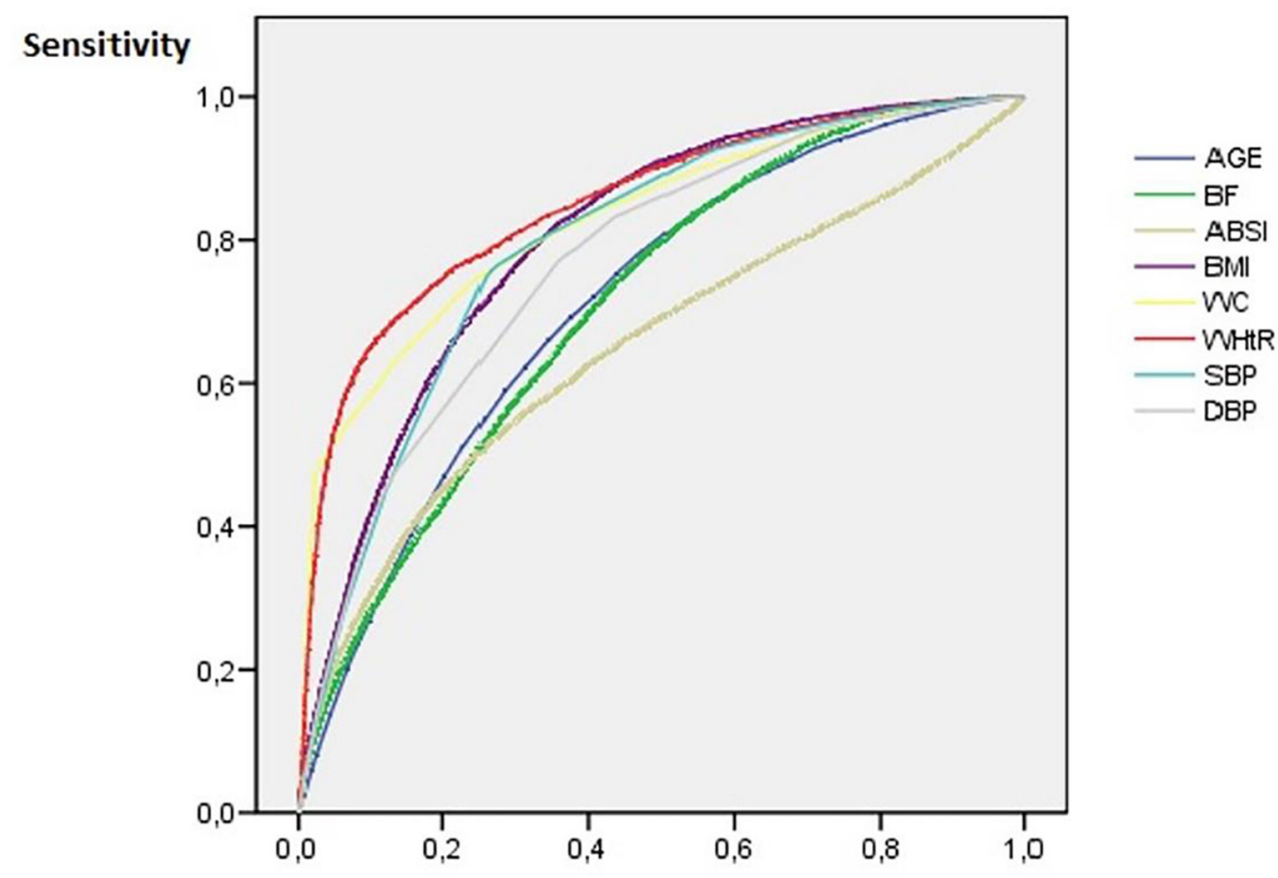

1-Specificity

\begin{tabular}{cccccc}
\hline AUC (95\% CI) & CP & $\begin{array}{c}\text { Sensitivity } \\
(\%)\end{array}$ & $\begin{array}{c}\text { Specificity } \\
(\%)\end{array}$ & JI \\
\hline Age & $0.71(0.71-0.72)$ & 42.5 & 69.2 & 62.5 & 0.32 \\
\hline WHtR & $0.85(0.84-0.86)$ & 0.54 & 68.5 & 87.0 & 0.56 \\
\hline WC & $0.83(0.82-0.84)$ & 89.1 & 72.5 & 77.6 & 0.50 \\
\hline BMI & $0.8(0.8-0.81)$ & 27.1 & 78.4 & 68.3 & 0.47 \\
\hline ABSI & $0.65(0.64-0.66)$ & 0.07 & 50.4 & 75.2 & 0.26 \\
\hline BF\% & $0.71(0.7-0.72)$ & 29.4 & 70.9 & 58.1 & 0.3 \\
\hline SBP & $0.79(0.79-0.8)$ & 127.5 & 75.8 & 73.5 & 0.49 \\
\hline DBP & $0.77(0.76-0.78)$ & 78.5 & 77.3 & 63.9 & 0.41 \\
\hline
\end{tabular}

Figure 1 Anthropometric variables. Receiver operating characteristic (ROC) curves, area under the curve (AUC), cut-off points and efficacy indicators. ABSI, A Body Shape Index; \%BF, percentage of body fat calculated according to the Deurenberg equation; BMI, body mass index; CP, cut-off point; DBP, diastolic blood pressure; JI, Youden Index; SBP, systolic blood pressure; WC, waist circumference; WHtR, waist-to-height ratio.

subjects with WHtR $\geq 0.56$ and $\mathrm{BP} \geq 128 / 80 \mathrm{~mm} \mathrm{Hg}$, would have a $50.5 \%$ risk of having MetS.

\section{DISCUSSION}

The validation of NIM-MetS was carried out from a study of diagnostic tests conducted in Spanish Caucasian adult workers and using, as a reference test, the NCEP-ATP III criteria for the diagnosis of MetS. The early detection of MetS is the key to improving the quality of life of our population, since it prevents the appearance of associated complications such as CVD, type 2 diabetes mellitus and, even, cancer. ${ }^{17-20}$

NIM-MetS has shown high VI in men $(88.6 \%)$ and women $(94.7 \%)$. Overall, for every 100 workers, the method properly classified 91 cases. Similarly, NIM-MetS has proved to be highly specific, reaching an overall SP of $94.9 \%$ (92.5\% in men and $97.8 \%$ in women). Both VI and the SP recorded values above those achieved by this method in another Spanish population, ${ }^{15}$ where it obtained an IV $=89.5 \%$ and $91.5 \%$ SP. For S, the overall figure was $56.4 \%$ ( $59.4 \%$ for men and $40.9 \%$ for women), while in the original population, the overall $\mathrm{S}$ was $77.9 \%$. Because it supposes a simple, easy to apply even in large populations and non-invasive method, it could be defined as a useful method in spite of the $\mathrm{S}$ found in the present study could be considered as moderate. The high SP together with the high VI shown for the screening of the cardiometabolic risk are characteristics that increase the acceptability of the method.

Although the indicators of validation and accuracy of a screening test (S and SP) are intrinsic properties of the test itself and do not depend on the prevalence of the disease considered, this does not prevent these indicators from being affected by characteristics of the population they are applied to. ${ }^{21}$ In fact, the most common observation is that a test for early detection or diagnosis alters its $\mathrm{S}$ and SP depending on these characteristic features of the population. Therefore, the main differences between the two populations (the Balearic and the one considered in the previous study developed in Cordoba ${ }^{15}$ ) were 


\begin{tabular}{|c|c|c|c|c|c|c|c|c|c|}
\hline \multirow{2}{*}{$\begin{array}{l}\text { WHtR } \\
\text { range } \\
\text { Cut-off } \\
\text { points } \\
\end{array}$} & \multirow{2}{*}{$\begin{array}{l}\text { BP model } \\
\text { BP }\end{array}$} & \multicolumn{4}{|c|}{$\begin{array}{l}\text { Probabilities of MetS for nodes in the decision } \\
\text { tree }\end{array}$} & \multicolumn{4}{|c|}{ Efficacy indicators for diagnostic test accuracy } \\
\hline & & Node 3 & Node 4 & Node 5 & Node 6 & $\begin{array}{l}\text { Sensitivity } \\
(\%)\end{array}$ & $\begin{array}{l}\text { Specificity } \\
(\%)\end{array}$ & VI (\%) & Jl \\
\hline \multirow[t]{3}{*}{0.535} & $\mathrm{BP}^{*}$ & 0.3 & 7.4 & 10.3 & 38.4 & 63.9 & 89.7 & 87.5 & 0.54 \\
\hline & $\mathrm{BP}+$ & 0.5 & 10.4 & 12.2 & 43.3 & 59.3 & 92.3 & 89.3 & 0.52 \\
\hline & SBP & 0.6 & 10.5 & 13.7 & 43.3 & 57.0 & 92.6 & 89.4 & 0.5 \\
\hline \multirow[t]{3}{*}{0.540} & $\mathrm{BP}^{*}$ & 0.3 & 7.6 & 11.5 & 40.7 & 62.7 & 90.9 & 88.4 & 0.54 \\
\hline & $\mathrm{BP}+$ & 0.5 & 10.5 & 13.5 & 45.7 & 57.8 & 93.2 & 90.0 & 0.51 \\
\hline & SBP & 0.6 & 10.7 & 15.2 & 45.7 & 55.6 & 93.5 & 90.0 & 0.49 \\
\hline \multirow[t]{3}{*}{$0.544 \ddagger$} & $\mathrm{BP}^{\star}$ & 0.4 & 7.7 & 12.5 & 42.8 & 61.6 & 91.8 & 89.1 & 0.53 \\
\hline & $\mathrm{BP}+$ & 0.5 & 10.9 & 14.6 & 48.0 & 56.7 & 93.9 & 90.5 & 0.51 \\
\hline & SBP & 0.6 & 10.8 & 16.4 & 48.0 & 54.5 & 94.1 & 90.6 & 0.49 \\
\hline \multirow[t]{3}{*}{$0.550 \S$} & $\mathrm{BP}^{\star}$ & 0.4 & 7.9 & 14.2 & 46.1 & 59.6 & 93.1 & 90.1 & 0.53 \\
\hline & BP† & 0.5 & 10.9 & 16.6 & 51.3 & 54.7 & 94.8 & 91.2 & 0.46 \\
\hline & SBP & 0.7 & 11.0 & 18.5 & 51.4 & 52.6 & 95.1 & 91.2 & 0.48 \\
\hline \multirow[t]{3}{*}{0.555} & $\mathrm{BP}^{\star}$ & 0.4 & 8.2 & 15.7 & 49.1 & 57.8 & 94.1 & 90.8 & 0.52 \\
\hline & $\mathrm{BP}+$ & 0.6 & 11.1 & 18.3 & 54.4 & 53.0 & 95.6 & 91.7 & 0.49 \\
\hline & SBP & 0.7 & 11.3 & 20.3 & 54.5 & 51.0 & 95.8 & 91.7 & 0.47 \\
\hline \multirow[t]{3}{*}{0.560} & $\mathrm{BP}^{\star}$ & 0.5 & 8.5 & 17.0 & 51.8 & 55.7 & 94.9 & 91.3 & 0.51 \\
\hline & $\mathrm{BP}+$ & 0.6 & 11.5 & 19.9 & 57.1 & 51.0 & 96.2 & 92.1 & 0.47 \\
\hline & SBP & 0.8 & 11.6 & 21.9 & 57.2 & 49.1 & 96.4 & 92.1 & 0.46 \\
\hline \multirow[t]{3}{*}{0.565} & $\mathrm{BP}^{*}$ & 0.5 & 8.8 & 18.6 & 54.9 & 53.4 & 95.6 & 91.8 & 0.49 \\
\hline & BP† & 0.6 & 11.9 & 21.8 & 60.3 & 48.8 & 96.8 & 92.5 & 0.46 \\
\hline & SBP & 0.8 & 12.0 & 23.9 & 60.4 & 47.0 & 96.9 & 92.4 & 0.44 \\
\hline \multirow[t]{3}{*}{0.570} & $\mathrm{BP}^{*}$ & 0.5 & 9.1 & 19.9 & 57.4 & 51.4 & 96.2 & 92.2 & 0.48 \\
\hline & $\mathrm{BP}+$ & 0.7 & 12.3 & 23.3 & 62.8 & 46.9 & 97.2 & 92.7 & 0.44 \\
\hline & SBP & 0.9 & 12.4 & 25.5 & 63.0 & 45.2 & 97.4 & 92.7 & 0.43 \\
\hline
\end{tabular}

*BP $\geq 128 / 80 \mathrm{~mm} \mathrm{Hg}$.

$\dagger B P \geq 128 / 85 \mathrm{~mm} \mathrm{Hg}$.

$\ddagger$ Cut-off point for WHtR obtained in the total simple $(n=60799)$.

§Cut-off point proposed by NIM-MetS.

BP, blood pressure; JI, Youden Index; NIM-MetS, non-invasive method for early detection of metabolic syndrome; SBP, systolic blood pressure $\geq 128 \mathrm{~mm} \mathrm{Hg}$; VI, Validity Index; WHtR, waist-to-height ratio.

analysed, highlighting those features of the Balearic population which contributed to a decreased S: a younger population (40.0 years vs 45.1 years), more women ( $42.7 \%$ vs $32.1 \%)$, more smokers $(34.8 \%$ vs $28.6 \%)$ and lower values for WC $(82.9 \mathrm{~cm}$ vs $87.8 \mathrm{~cm})$, WHtR $(0.49 \mathrm{vs}$ $0.52)$ and BMI $\left(26.1 \mathrm{~kg} / \mathrm{m}^{2}\right.$ vs $\left.26.5 \mathrm{~kg} / \mathrm{m}^{2}\right)$.

As regards the safety indicators of the test, the PPV and $\mathrm{NPV}$, they are definitely affected by the prevalence of the population, lowering the PPV when the prevalence of MetS is lower. In this way, although we found a lower prevalence of MetS in the Balearic Islands than in Cordoba $(9.0 \%$ vs $13.9 \%)$, the NIM-Mets produced a lower PPV in the Balearic Islands (51.3\% vs $61.7 \%)$, while the NPV remained very similar $(95.5 \%$ vs $95.9 \%)$.

Screening tests are often used in clinical practice. However, there are very few methods for the early detection of MetS other than the currently known diagnostic criteria, and there are even fewer non-invasive screening tests. A study in the Republic of Korea examined the validity of a test for the early detection of MetS based on the muscle-to-fat ratio. ${ }^{22}$ The study was conducted on 6256 participants, with an $\mathrm{S}$ of $68.6 \%$ in men and $76.0 \%$ in women, and an SP of $63.8 \%$ in men and $53.8 \%$ in women. Miller et $a l^{23}$ proposed a screening method for MetS in 745 young adults (18-29 years old) in USA, based on making decision trees with the CHAID methodology and using all the criteria for MetS. The method had a validity rate of $89.4 \%$ and an S rate of $61.7 \%$. Finally, De Kroon $e t a l^{24}$ conducted a screening test for MetS in 642 young people (aged 17-28 years) in the Netherlands using anthropometric variables (BMI, WC and BP). The $\mathrm{S}$ of the method was $68.7 \%$ and the VI was $95.6 \%$. 
0.6

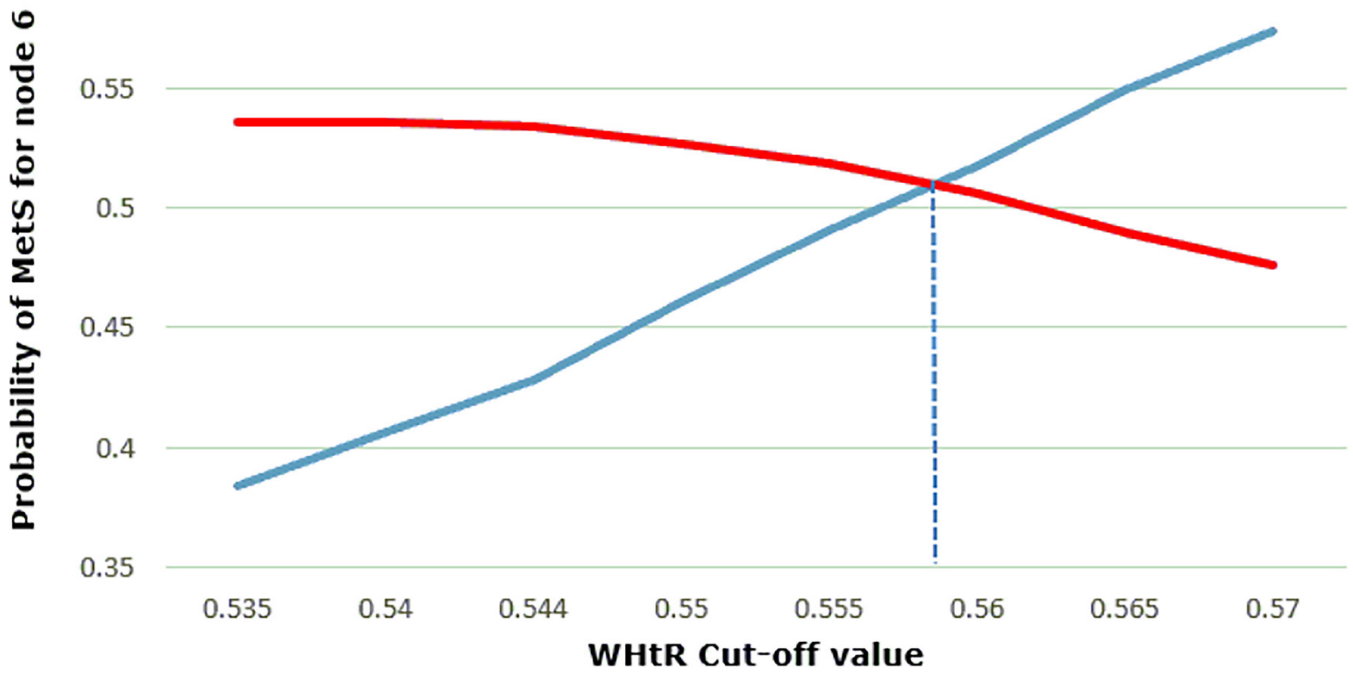

Figure 2 Waist-to-height ratio (WHtR) cut-off point resolution. MetS, metabolic syndrome.

Another hypothesis put forward in this research was to test whether the cut-off value proposed by NIM-MetS for WHtR $(\geq 0.55)$ would be reproduced in a large sample (60 799 workers from the Balearic Islands), and if it was also valid for both men and women and also for different age groups. WHtR had a cut-off value of 0.54 for the total sample, with 0.56 men and 0.53 for women. As regards age groups, the WHtR threshold increased with age, with $0.55-0.56$ for men and $0.51-0.55$ for women. These differences were reduced in the total sample $(0.53-0.56)$.

It is noteworthy that several authors have proposed a universal 0.50 cut-off point for WHtR, both to detect MetS and to predict cardiometabolic risk and overall cardiovascular mortality. ${ }^{25-28}$ However, in Spain, a cross-sectional 
study in the general population $(n=6279$, mean age $=43$ years) showed that WHtR was the best anthropometric predictor of MetS (NCEP-ATP III), and the authors proposed a cut-off value of 0.55 , with which they obtained an S of $91.0 \%$ and an SP of $64.0 \% .^{28}$ This cut-off value is very similar to the one proposed from the results obtained in the present study.

In Chile, two important child population studies were conducted by Arnaiz et al, showing results which match the value of the cut-off point proposed in the present study. Thus, in the first study, conducted on 209 schoolchildren (mean age of 11.5 years), the authors obtained a cut-off value of $0.55 \mathrm{WHtR}$ for MetS, ${ }^{29}$ while in the second study, performed in 2980 children aged $6-14$ years (mean age of 9.9), the authors concluded that the WHtR did not change with age and gender and, therefore, a universal cut-off value could be agreed on for both children and adults. $^{30}$

The prospective study by Koch $e t a l^{\beta 1}$ carried out in Chile on about 6714 men and 6340 women, evaluating the relationship between various anthropometric indices of adiposity, cardiovascular risk factors and mortality for a cut-off value of 0.55 obtained an S of $75.8 \%$ and SP of $73.3 \%$ for men, and an S of $77.6 \%$ and SP of $56.3 \%$ for women.

In addition, several investigations conducted in non-European and non-Hispanic populations also concur on this threshold of WHtR for MetS. Thus, Obeidat et $a l^{2}$ in a study on a Jordanian population $(n=630$, aged $20-70$ years) reported a cut-off value of 0.56 in men and 0.52 in women; in India, Rajput $e t a l,{ }^{33}$ achieved a threshold of 0.52 for men and women $(\mathrm{n}=3042)$ in all locations (rural or urban areas); and in China, He et al, ${ }^{34}$ in a descriptive study of 1068 adult subjects, reported a cut-off value for WHtR of 0.5 for men and women alike.

When the NIM-MetS method was applied in this new larger sample of 60799 workers from the Balearic Islands, the method has showed again the same variables obtained in the original study performed in Cordoba. ${ }^{15}$ In the multiple logistic regression, WHtR and BP achieved the highest adjusted OR values. Thus, WHtR was the anthropometric index that best discriminated MetS presence, with an adjusted OR value of 4.4 (3.9-4.9), while SBP obtained an adjusted OR value of 3.8 (3.5-4.1). In addition, the cut-off values obtained for WHtR and for BP are very similar to those of the original method.

Several investigations have confirmed the high predictive ability of WHtR for MetS and CVD. In the systematic review conducted by Ashwell et al, ${ }^{35}$ in which 10 out of the 31 studies analysed the association between anthropometric measurements of central obesity and MetS, WHtR had the highest AUC value of 0.76 (men) and 0.75 (women). This contrasted with WC, which obtained an AUC value of 0.75 (equal for men and women) and BMI, with an AUC value of 0.72 (men and women). Similarly, a meta-analysis conducted by Savva $e t a l,{ }^{36}$ in which 8 out of the 24 studies included compared WHtR (cut-off point 0.5 ) with BMI (cut-off point of 23 for the Asian population and 25 for the rest) for cardiometabolic risk in Asian and non-Asian populations, concluded that WHtR showed a stronger association with MetS than with BMI.

Through the present study, the NIM-MetS method has been reproduced, and definitive cut-off values have been proposed for WHtR (0.56) and BP (128-80 $\mathrm{mm} \mathrm{Hg})$, from which an S rate of $56.4 \%$, an SP rate of $94.5 \%$, a VI of $91.1 \%$ and a JI of 0.51 are obtained. On the other hand, finally, the long-term ability of MetS to predict CVD has been shown to be limited by the dichotomous (binary) and qualitative nature of the classic diagnostic criteria for MetS. An innovative aspect that NIM-MetS brings is to provide a gradient or scale of risk of developing MetS which is divided into four risk levels: very low risk $(\mathrm{p}=0.4 \%)$, low risk $(\mathrm{p}=8.3 \%)$, moderate risk $(\mathrm{p}=16.4 \%)$ and high risk $(\mathrm{p}=50.5 \%)$. In this way, health professionals can take certain steps depending on the level of risk of MetS and promote a more accurate and early detection of the possible complications associated with CVD and MetS. Along the same lines, there have been several studies using methods based on scores to quantify the amount of risk accumulated by the presence of the components that define the MetS (Metabolic Syndrome Severity Score) ${ }^{37} 38$

\section{Limitations}

This study presents some limitations that must be acknowledged. First, we must bear in mind that there are different definitions and criteria to determine the presence of MetS. In this study, the presence of MetS was ascertained using the NCEP-ATP III definition as the gold standard, which supposes one of the definitions most used and widely accepted by the international community and the WHO. In addition, the study data refer to the Caucasian population. Thus, results could not have great applicability to other populations.

Although in the present study NIM-MetS methodology has been tested in a very large sample of workers, the $\mathrm{S}$ found was lower than that obtained in the original study leading to the proposal of the method. ${ }^{15}$ This could be related to differences in the study samples, with the workers from the Balearic Islands showing lower prevalence of SMet and obesity and being younger. Although the prevalence of MetS does not affect $\mathrm{S}$ and SP, this lower prevalence influences PPV and NPV.

Although the percentage of participation is high $(87.4 \%)$, we should take into account that it is not the total target population and, therefore, a bias could have been introduced in the results. Furthermore, participants highly concerned about their health, and thus probably healthier, along with those with a diagnosed disease, could represent the greater proportion of workers attending health examinations because these were not compulsory. This causes bias in the recruitment procedure as, in addition, it is not well known whether the healthier workers or the ones with a diagnosed disease are the ones with the greatest interest in the checks. Nor can we ignore the bias of the healthy worker, 
since those workers with serious illnesses would not be currently active. In addition, it is not well known if the healthiest workers or those with a diagnosed illness have the greatest interest in checks.

\section{CONCLUSIONS}

NIM-MetS has proved to be a valid method for the early detection of MetS in a healthy worker population. It is a simple, economical and quick non-invasive test which is easy to apply and interpret in any healthcare setting (primary healthcare, hospitals, occupational health) as well as in other settings (education, sport, etc). WHtR is the best predictor of MetS and its cut-off point can be used for both genders and for different age groups. The clinical decision tree that produces NIM-MetS uses WHtR (0.56) and BP (128/80 mm Hg), and obtains high SP and diagnostic validity. NIM-MetS provides a gradient or risk scale which allows a more accurate and earlier detection of CVD in subjects with risk of MetS.

\section{Author affiliations \\ ${ }^{1}$ Department of Occupational Safety and Health, Córdoba City Hall, Córdoba, Spain ${ }^{2}$ Department of Fundamental Biology and Health Sciences, University of the Balearic Islands, Palma, Spain \\ ${ }^{3}$ Research Group on Evidence, Lifestyles \& Health, Research Institute on Health Sciences (IUNICS), University of the Balearic Islands, Palma, Spain \\ ${ }^{4}$ Department of Occupational Risk Prevention and Environmental Protection, University of Córdoba, Córdoba, Spain \\ ${ }^{5}$ Prevention of Occupational Risks in Health Services, Balearic Islands Health Service, Palma, Spain \\ ${ }^{6}$ IMIBIC, Reina Sofía University Hospital, University of Córdoba, Córdoba, Spain ${ }^{7}$ CIBER Physiopathology of Obesity and Nutrition CIBEROBN, ISCIII, Madrid, Spain ${ }^{8}$ Nursing and Physiotherapy Department, University of the Balearic Islands, Palma, Spain \\ ${ }^{9}$ Department of Nursing, School of Medicine and Nursing, University of Córdoba, Córdoba, Spain}

Contributors MR-S and contributed tothe conception, design, acquisition and analysis/interpretation of data, and draftedthe manuscript. PT contributed to the data collection and analysis. MV-Acontributed to analysis and interpretation of data. A-AL-G contributed to thedata collection and analysis. F-JF-J contributed to the analysis of data. AA andCA-F contributed to the conception. GM-R contributed to the analysis andinterpretation. MB-V contributed to the design, acquisition andanalysis/ interpretation of data. All authors critically revised the manuscript,gave final approval and agree to be accountable for the integrity and accuracyof all aspects of the work.

Funding The authors have not declared a specific grant for this research from any funding agency in the public, commercial or not-for-profit sectors.

Competing interests None declared.

Patient consent Not required.

Ethics approval Institutional Review Board of the Mallorca Health Management Ethical Review Committee of GESMA.

Provenance and peer review Not commissioned; externally peer reviewed.

Data sharing statement Extra data can be accessed via the Dryad data repository at http://datadryad.org/ with the doi:10.5061/dryad.cb51t54.

Open access This is an open access article distributed in accordance with the Creative Commons Attribution Non Commercial (CC BY-NC 4.0) license, which permits others to distribute, remix, adapt, build upon this work non-commercially, and license their derivative works on different terms, provided the original work is properly cited, appropriate credit is given, any changes made indicated, and the use is non-commercial. See: http://creativecommons.org/licenses/by-nc/4.0/.

\section{REFERENCES}

1. Swinburn BA, Sacks G, Hall KD, et al. The global obesity pandemic: shaped by global drivers and local environments. Lancet 2011;378:804-14.

2. Tauler P, Bennasar-Veny M, Morales-Asencio JM, et al. Prevalence of premorbid metabolic syndrome in Spanish adult workers using IDF and ATPIII diagnostic criteria: relationship with cardiovascular risk factors. PLoS One 2014;9:e89281.

3. Yamaoka K, Tango T. Effects of lifestyle modification on metabolic syndrome: a systematic review and meta-analysis. BMC Med 2012;10:138

4. Guh DP, Zhang W, Bansback N, et al. The incidence of comorbidities related to obesity and overweight: a systematic review and meta-analysis. BMC Public Health 2009:9:88.

5. Schneider HJ, Klotsche J, Silber S, et al. Measuring abdominal obesity: effects of height on distribution of cardiometabolic risk factors risk using waist circumference and waist-to-height ratio. Diabetes Care 2011;34:e7.

6. Zimmet PZ, Alberti KG, Shaw JE. Mainstreaming the metabolic syndrome: a definitive definition. Med J Aust 2005;183:175-6.

7. Bennasar-Veny M, Lopez-Gonzalez AA, Tauler P, et al. Body adiposity index and cardiovascular health risk factors in Caucasians: a comparison with the body mass index and others. PLoS One 2013;8:e63999.

8. Rosales M. Antropometría en el diagnóstico de pacientes obesos: una revisión. Nutr Hosp 2012;27:1803-9.

9. Edston E. A correlation between the weight of visceral adipose tissue and selected anthropometric indices: an autopsy study. Clin Obes 2013;3:84-9.

10. Kodama S, Horikawa C, Fujihara K, et al. Comparisons of the strength of associations with future type 2 diabetes risk among anthropometric obesity indicators, including waist-to-height ratio: a meta-analysis. Am J Epidemiol 2012;176:959-69.

11. Petursson $\mathrm{H}$, Sigurdsson JA, Bengtsson $\mathrm{C}$, et al. Body configuration as a predictor of mortality: comparison of five anthropometric measures in a 12 year follow-up of the Norwegian HUNT 2 study. PLoS One 2011;6:e26621.

12. Taylor AE, Ebrahim S, Ben-Shlomo Y, et al. Comparison of the associations of body mass index and measures of central adiposity and fat mass with coronary heart disease, diabetes, and all-cause mortality: a study using data from 4 UK cohorts. Am J Clin Nutr 2010;91:547-56.

13. Lam BC, Koh GC, Chen C, et al. Comparison of Body Mass Index (BMI), Body Adiposity Index (BAI), Waist Circumference (WC), WaistTo-Hip Ratio (WHR) and Waist-To-Height Ratio (WHtR) as predictors of cardiovascular disease risk factors in an adult population in Singapore. PLoS One 2015;10:e0122985.

14. Hsieh SD, Yoshinaga $\mathrm{H}$. Waist/height ratio as a simple and useful predictor of coronary heart disease risk factors in women. Intern Med 1995;34:1147-52.

15. Romero-Saldaña M, Fuentes-Jiménez FJ, Vaquero-Abellán M, et al. New non-invasive method for early detection of metabolic syndrome in the working population. Eur J Cardiovasc Nurs 2016;15:549-58.

16. Stewart A, Marfell-Jones M, Olds T, et al. International standards for anthropometric assessment. Lower Hutt, New Zealand: ISAK, 2011.

17. Braun S, Bitton-Worms K, LeRoith D. The link between the metabolic syndrome and cancer. Int J Biol Sci 2011;7:1003-15.

18. Devers MC, Campbell S, Simmons D. Influence of age on the prevalence and components of the metabolic syndrome and the association with cardiovascular disease. BMJ Open Diabetes Res Care 2016;4:e000195.

19. Guo Y, Musani SK, Sims M, et al. Assessing the added predictive ability of a metabolic syndrome severity score in predicting incident cardiovascular disease and type 2 diabetes: the Atherosclerosis Risk in Communities Study and Jackson Heart Study. Diabetol Metab Syndr 2018;10:42

20. Gami AS, Witt BJ, Howard DE, et al. Metabolic syndrome and risk of incident cardiovascular events and death: a systematic review and meta-analysis of longitudinal studies. J Am Coll Cardiol 2007;49:403-14.

21. Brenner $H$, Gefeller O. Variation of sensitivity, specificity, likelihood ratios and predictive values with disease prevalence. Stat Med 1997;16:981-91.

22. Park J, Kim S. Validity of muscle-to-fat ratio as a predictor of adult metabolic syndrome. J Phys Ther Sci 2016;28:1036-45.

23. Miller B, Fridline M, Liu PY, et al. Use of CHAID decision trees to formulate pathways for the early detection of metabolic syndrome in young adults. Comput Math Methods Med 2014;2014:1-7.

24. de Kroon ML, Renders CM, Kuipers EC, et al. Identifying metabolic syndrome without blood tests in young adults-the Terneuzen Birth Cohort. Eur J Public Health 2008;18:656-60. 
25. Ashwell M, Hsieh SD. Six reasons why the waist-to-height ratio is a rapid and effective global indicator for health risks of obesity and how its use could simplify the international public health message on obesity. Int J Food Sci Nutr 2005;56:303-7.

26. Bellido D, López de la Torre M, Carreira J, et al. [Anthropometric measures of central abdominal fat and discriminant capacity for metabolic syndrome in a Spanish population]. Clin Investig Arterioscler 2013;25:105-9.

27. Browning LM, Hsieh SD, Ashwell M. A systematic review of waist-toheight ratio as a screening tool for the prediction of cardiovascular disease and diabetes: 0.5 could be a suitable global boundary value. Nutr Res Rev 2010;23:247-69.

28. Cristo Rodríguez Pérez MD, Cabrera De León A, Aguirre-Jaime A, et al. [The waist to height ratio as an index of cardiovascular risk and diabetes]. Med Clin 2010;134:386-91.

29. Arnaiz P, Marín A, Pino F, et al. [Waist height ratio, ultrasensitive $c$ reactive protein and metabolic syndrome in children]. Rev Med Chil 2010;138:1378-85.

30. Arnaiz P, Grob F, Cavada G, et al. [Waist-to-height ratio does not change with gender, age and pubertal stage in elementary school children]. Rev Med Chil 2014:142:574-8.

31. Koch E, Romero T, Manríquez L, et al. Razón cintura-estatura: Un mejor predictor antropométrico de riesgo cardiovascular y mortalidad en adultos chilenos. Nomograma diagnóstico utilizado en el Proyecto San Francisco. Rev Chil de Cardiol 2008;27:23-35.
32. Obeidat AA, Ahmad MN, Haddad FH, et al. Evaluation of several anthropometric indices of obesity as predictors of metabolic syndrome in Jordanian adults. Nutr Hosp 2015;32:667-77.

33. Rajput R, Rajput M, Bairwa M, et al. Waist height ratio: a universal screening tool for prediction of metabolic syndrome in urban and rural population of Haryana. Indian J Endocrinol Metab 2014;18:394-9

34. He YH, Chen YC, Jiang GX, et al. Evaluation of anthropometric indices for metabolic syndrome in Chinese adults aged 40 years and over. Eur J Nutr 2012;51:81-7.

35. Ashwell M, Gunn P, Gibson S. Waist-to-height ratio is a better screening tool than waist circumference and BMI for adult cardiometabolic risk factors: systematic review and meta-analysis. Obes Rev 2012;13:275-86.

36. Savva SC, Lamnisos D, Kafatos AG. Predicting cardiometabolic risk: waist-to-height ratio or BMI. A meta-analysis. Diab Metab Syndr Obes 2013;6:403-19.

37. DeBoer MD, Gurka MJ, Woo JG, et al. Severity of the metabolic syndrome as a predictor of type 2 diabetes between childhood and adulthood: the Princeton lipid research cohort study. Diabetologia 2015;58:2745-52.

38. Wiley JF, Carrington MJ. A metabolic syndrome severity score: a tool to quantify cardio-metabolic risk factors. Prev Med 2016;88:189-95. 